REVISTA MATEMATICA de la

Universidad Complutense de Madrid

Volumen 6, número 2; 1993

http://dx.doi.org/10.5209/rev_REMA.1993.v6.n2.17824

\title{
Local Connectivity, Open Homogeneity and Hyperspaces
}

\author{
J. J. Charatonik
}

ABSTRACT. In the first part of the paper behaviour of conditions related to local connectivity at a point is discussed if the space is transformed under a mapping that is interior or open at the considered point of the domain. The second part of the paper deals with metric locally connected continua. They are characterized as continua for which the hyperspace of their nonempty closed subsets is homogeneous with respect to open mappings. A similar characterization for the hyperspace of subcontinua remains an open question.

Topological spaces considered in this paper are assumed to be Hausdorff, and mappings are assumed to be continuous. A continuum means a compact connected space.

\section{LOCAL CONNECTIVITY AND MAPPINGS}

A space $X$ is said to be connected im kleinen at a point $p$ if for each open subset $A$ of $X$ such that $p \in A$ there exists an open subset $B$ of $X$ such that $p \in B$ and $B$ is contained in a component of $A$ ([11], p. 89). In other words, a space $X$ is connected im kleinen at $p \in X$ provided that for each open set $A$ containing $p$ there is an open set $B$ containing $p$ and lying in $A$ such that for each point $q \in B$ there is a connected subset of $A$ containing both $p$ and $q$ ([4], p. 113); equivalently, if for each open neighbourhood $A$ of $p$ the point $p$ is an interior point of a component of $A$.

1991 Mathematics Subject Classification: 54B20, 54C50, 54F15.

Editorial Complutense. Madrid, 1993. 
A space $X$ is said to be locally connected at $p \in X$ provided that for each open subset $A$ of $X$ such that $p \in A$ there is a connected open subset $B$ of $X$ such that $p \in B \subset A$; equivalently, if $X$ has a local basis at $p$ composed of connected open sets $([11]$, p. $89 ;[4]$, p. 105). Note that Kuratowski uses the term «locally connected at a point» in the sense of connectivity im kleinen at this point, see the definition in [7], $\$ 49, I$, p. 227.

Obviously, by the definitions

(1) if a space is locally connected at a point $p$, then it is connected im kleinen at $p$,

but not inversely. Well-known examples of plane continua which are connected im kleinen but not locally connected at a point are presented e.g. in [4], Fig. 3-9, p. 113 and [5], Example 1, p. 137.

A space is said to be connected im kleinen (locally connected) provided it is connected im kleinen (locally connected) at each of its points. It is known (see e.g. [11], Theorem 10, p. 90) that

(2) if a space is connected im kleinen at every point of some open set that contains a point $p$, then it is locally connected at $p$. that

In particular, it follows from (2) (see e.g. [4], Theorem 3-11, p. 114)

(3) if a space is connected im kleinen (at each of its points), then it is locally connected.

A mapping $f: X \rightarrow Y$ is said to be interior at a point $p \in X$ provided for every open neighbourhood $U$ of $p$ in $X$ the point $f(p)$ is in the interior of $f(U)$. A mapping is said to be open if it maps open subsets of the domain to open subsets of the range. Obviously

(4) a mapping is open if and only if it is interior at each point of its domain. 
5. Proposition. Let a space $X$ be connected im kleinen at a point $p$. If a mapping $f$ defined on $X$ is interior at $p$, then $f(X)$ is connected im kleinen at $f(p)$.

Proof. Let $V$ be an open set in $f(X)$ with $f(p) \in V$. Since $f$ is continuous, $f^{-1}(V)$ is an open subset of $X$ containing $p$. By connectedness im kleinen of $X$ at $p$ there is an open subset $U$ of $X$ such that $p \in U$ and $U$ is contained in a component $C$ of $f^{-1}(V)$. Since $f$ is interior at $p$, we have $f(p) \in$ int $f(U) \subset f(U) \subset f(C) \subset V$. Thus int $f(U)$ is an open subset of $f(X)$ that is contained in a connected subset $f(C)$ of $V$. The proof is complete.

Statement (1) and Proposition 5 imply the following.

6. Corollary. Let a space $X$ be locally connected at a point $p$. If a mapping $f$ defined on $X$ is interior at $p$, then $f(X)$ is connected im kleinen at $f(p)$.

Conclusion of Corollary 6 cannot be strengthened by saying that $f(X)$ is locally connected at $f(p)$. The author is obliged to $\mathrm{K}$. Omiljanowski and J. R. Prajs for calling to his attention the following example showing the statement. We recall some definitions first. A space is said to be rational if it has an open basis with countable boundaries. A dendroid means an arcwise connected and hereditarily unicoherent (metric) continuum. The cone over the closure of the harmonic sequence $\{0\} \cup\{1 / n: n \in \mathbb{N}\}$ is called the harmonic fan. Note that the harmonic fan is a dendroid. An arc $a b$ contained in a space $S$ is said to be free provided $a b \backslash\{a, b\}$ is an open subset of $S$. A mapping with connected point-inverses is defined to be monotone.

7. Example. There exist rational plane dendroids $X$ and $Y$, a point $P$ of $X$ and a surjective mapping $f: X \rightarrow Y$ such that $f$ is monotone and interior at $p$, the dendroid $X$ is locally connected at $p$, while $Y$ is not locally connected at $f(p)$.

Proof. Put, in the Cartesian coordinates in the plane, $p=(0,0)$, and, for each positive integer $n$, let

$$
\begin{array}{ll}
a_{n}=\left(2^{-n-1}, 0\right), & a_{n, m}=\left(2^{-n-1}, 2^{-m-n-1}\right), \\
b_{n}=\left(3 \cdot 2^{-n-3}, 0\right), & b_{n, m}=\left(3 \cdot 2^{-n-3}, 3 \cdot 2^{-m-n-3}\right) .
\end{array}
$$


Therefore $b_{n}$ is the middle point of $a_{n+1}$ and $a_{n}$. Denoting by $a b$ the straight line segment joining points $a$ with $b$ in the plane, put

$$
X=p a_{1} \cup \cup\left\{\cup\left\{a_{n} b_{n, m}: m \in \mathbb{N}\right\}: n \in \mathbb{N}\right\}
$$

and

$$
Y=p a_{1} \cup \cup\left\{\bigcup\left\{a_{n} a_{n+1, m}: m \in \mathbb{N}\right\}: n \in \mathbb{N}\right\} .
$$

Hence $X$ and $Y$ are rational plane dendroids simply by their constructions. The dendroid $Y$ is homeomorphic to the above mentioned example pictured in [4], Fig. 3-9, p. 113. Observe that $X$ is locally connected at $p$, while $Y$ is connected im kleinen, but not locally connected at $p$.

Note that for each $n \in \mathbb{N}$ the unions

$$
a_{n} b_{n} \cup \bigcup\left\{a_{n} b_{n, m}: m \in \mathbb{N}\right\} \text { and } a_{n} a_{n+1} \cup \cup\left\{a_{n} a_{n+1, m}: m \in \mathbb{N}\right\},
$$

which are contained in $X$ and $Y$ respectively, are homeomorphic to a harmonic fan, and that the segments $a_{n+1} b_{n}$ are free $\operatorname{arcs}$ in $X$, while in the dendroid $Y$ there is no free arc contained in the segment $p a_{1}$.

In the dendroid $X$ shrink every free arc lying in the segment $p a_{1}$ to a point, and let $q: X \rightarrow q(X)$ stand for the quotient mapping. Note that $q$ is monotone and interior at $p$ and that $q(X)$ is homeomorphic to $Y$. Let $h: q(X) \rightarrow Y$ be a homeomorphism. Then it is enough to define $f=h q$. The proof is complete.

Applying a general notion of localization of a mapping (see [8], Chapter 4, C, p. 18) to the class of open mappings we get the following definition. A mapping $f: X \rightarrow Y$ between spaces $X$ and $Y$ is said to be $l o$ cally open at a point $p \in X$ provided that there exists a closed neighbourhood $U$ of $p$ such that $f(U)$ is a closed neighbourhood of $f(p)$ and the partial mapping $f \mid U: U \rightarrow f(U)$ is open. The following implication is known (see [1], Statement 13, p. 360).

(8) If a mapping is locally open at a point of its domain, then it is interior at this point.

The converse implication does not hold. Really, the mapping $f$ defined in Example 7 is interior at the point $p \in X$, while it is easy to note that there is no open neighbourhood $U$ of $p$ in $X$ with an open partial mapping $f \mid U: U \rightarrow f(U)$. Another example is described in [1], p. 360 . 
Similarly to (4) we have the following assertion.

(9) A mapping is open if and only if it is locally open at each point of its domain.

As it was said above, the concept of local cunnectivity at a point is not preserved under mappings which are interior at the considered point. But if the mapping is assumed to satisfy a stronger condition, namely to be locally open at the point, then the invariance takes place. Indeed, we have the following proposition.

10. Proposition. Let a space $X$ be locally connected at a point $p$. If a mapping $f$ defined on $X$ is locally open at $p$, then $f(X)$ is locally connected at $f(p)$.

As an immediate consequence of (9) and Proposition 10 we get a corollary.

11. Corollary. Let a space $X$ be locally connected at a point $p$. If a mapping $f$ defined on $X$ is open, then $f(X)$ is locally connected at $f(p)$.

\section{OPEN HOMOGENEITY}

A space $X$ is said to be openly homogeneous (homogeneous) provided for each its points $p$ and $q$ there is an open mapping (a homeomorphism) of $X$ onto itself that maps $p$ onto $q$. As an easy consequence of this definition and of Corollary 11 we have the next corollary.

12. Corollary. If a space is openly homogeneous and locally connected at some point, then it is locally connected.

Given a (Hausdorff) continuum $X$, we denote by $2^{x}$ and $C(X)$ the hyperspaces of all nonempty closed subsets and of all nonempty subcontinua of $X$, with the Vietoris topology, respectively.

13. Lemma. If, for a Hausdorff continuum $X$, either $2^{X}$ or $C(X)$ is openly homogeneous, then $X$ is locally connected. 
Proof. It is known that $2^{X}$ and $C(X)$ are both continua ([10], p. 1209) which are locally connected at $X([12],(1.136)$, p. 154). Thus, if one of the hyperspaces is openly homogeneous, it is locally connected by Corollary 12. This is known to be equivalent to local connectedness of $X$ ([12], (1.92), p. 134 and (1.208), p. 199).

14. Remark. For the metric case and under a stronger assumption of homogeneity of either $2^{X}$ or $C(X)$ the above lemma was shown in [3], p. 1032.

15. Remark. It was observed in [3], p. 1032 that $C(P)$ is not homogeneous for a pseudo-arc $P$. It follows from Lemma 13 that neither of the two hyperspaces of a pseudo-arc is openly homogeneous. However, a stronger result holds true.

16. Theorem. The following statements are equivalent for a nondegenerate metric continuum $X$ :

(i) $2^{x}$ is homogeneous;

(ii) $2^{x}$ is openly homogeneous;

(iii) $X$ is locally connected;

(iv) $2^{X}$ is homeomorphic to the Hilbert cube.

Proof. Trivially (i) implies (ii). By Lemma 13, (ii) implies (iii). Now (iii) is known to be equivalent to (iv) ([2], Theorem 1, p. 927), which implies (i) by [6].

17. Remark. Equivalence of conditions (i), (iii) and (iv) is shown in [12], (17.3), p. 565.

18. Remark. The example of an extended long line shows that metrizability is an essential assumption in Theorem 16 (see [12], (17.4), p. 565).

The concept of a free arc recalled here in the previous part of the paper has been used by S. B. Nadler, Jr., in [12], (17.2), p. 564 to characterize metric continua $X$ with homogeneous hyperspace $C(X)$. 
19. Theorem (Nadler). The following statements are equivalent for a non-degenerate metric continuum $X$ :

(i) $C(X)$ is homogeneous;

(ii) $X$ is locally connected and contains no free arc;

(iii) $C(X)$ is homeomorphic to the Hilbert cube.

20. Remark. Unlikely for the hyperspace $2^{X}$ in Theorem 16 , the condition saying that $C(X)$ is openly homogeneous cannot be joined to ones of the above theorem. Indeed, if $X$ is a metric arc, then $C(X)$ is homeomorphic to a disc, which is locally connected plane continuum, and hence not homogeneous (because a simply closed curve is the only such continuum, [9], p. 137), while known to be openly homogeneous by a recent result of J. R. Prajs ([13], Corollary 5).

21. Problem. Characterize metric continua $X$ for which the hyperspace $C(X)$ is openly homogeneous.

\section{References}

[1] J. J. Charatonik and K. Omilsanowski: On the set of interiority of a mapping, Glasnik Mat. 17 (37) (1982), 341-361.

[2] D. W. CuRTIS and R. M. SCHORI: $2^{X}$ and $C(\mathrm{X})$ are homeomorphic to the Hilbert cube, Bull. Amer. Math. Soc. 80 (1974), 927-931.

[3] C. EBERHART and S. B. NADLER, Jr.: The dimension of certain hyperspaces, Bull. Acad. Polon. Sci. Ser. Sci. Math. Astronom. Phys. 19 (1971), 1027-1034.

[4] J. G. Hocking and G. S. Young: Topology, Addison-Wesley Publ. Co. 1961.

[5] F. B. Jones: Concerning aposyndetic and non-aposyndetic continua, Bull. Amer. Math. Soc. 58 (1952), 137-151.

[6] O. H. KelleR: Die Homoiomorphie der kompakten konvexen Mengen in Hilbertschen Raum, Math. Ann. 105 (1931), 748-758.

[7] K. KURATowski: Topology, vol. 2, Academic Press and PWN, 1968.

[8] T. MACKOWIAK: Continuous mappings on continua, Dissertationes Math. (Rozprawy Mat.) 158 (1979), 1-91.

[9] S. MazurKIEWICZ: Sur les continua homogènes, Fund. Math. 5 (1924), 137-146.

[10] M. M. MCWATERS: Arcs, semigroups, and hyperspaces, Canad. J. Math. 20 (1968), 1207-1210.

[11] R. L. MoORE: Foundations of point set theory, Amer. Math. Soc. Colloq. Publ. Vol. 13, Amer. Math. Soc. 1962.

[12] S. B. NADLER, Jr.: Hyperspaces of sets, Dekker 1978. 
[13] J. R. PRAJS: On open homogeneity of closed balls in the Euclidean spaces (preprint).

Mathematical Institute

University of Wroclaw

Pl. Grunwaldzki 2/4

50-384 Wroclaw

Poland

Recibido: 19 de octubre de 1992

Revisado: 22 de marzo de 1993 